\title{
Histoplasmosis diseminada y Bartonellosis humana, reporte de un caso.
}

\author{
Disseminated Histoplasmosis and Bartonellosis. A case report
}

\author{
MAGUIÑA Ciro*,***, ACOSTA Raúl**, INDACOCHEA Sonia**, \\ BUSTAMANTE Beatriz $* * *$, SALINAS César****, COK Jaime ****, VARELA \\ Luis **, LOPEZ Marga**, SALMAVIDES Frine** MAC RAE John**,***.
}

*Centro de Investigación "Hugo Lumbreras". Instituto Nacional de Salud.

**Departamento de Medicina. Hospital Nacional Cayetano Heredia.

***Instituto de Medicina Tropical "Alexander Von Humboldt. Universidad Peruana Cayetano Heredia.,

****Departamento de Patología Hospital Nacional Cayetano Heredia.

\section{PRESENTACION DEL CASO CLINICO}

Agricultor de 19 años de edad, nacido y residente en Cabana (Ancash) y procedente de Tocache (Tingo María), ingresa al Hospital Nacional Cayetano Heredia (HNCH) el día 21-11-91, con un tiempo de enfermedad de 14 días inicia con lumbalgia intensa, cefalea global, dolores osteomusculares generalizados, fiebre no cuantificada, sudoración, malestar general, palidez progresiva, astenia adinamia, luego se agrega disnea a mediados y pequeños esfuerzo; vértigo y pérdida de conocimiento en 3 ocasiones. Viaje a Tocache 1 semana antes de su ingreso al hospital, donde diagnostican "fiebre tifoidea" recibiendo cloramfenicol por 3 días, al no mejorar decide acudir a Lima, donde es hospitalizado. Tenía apetito disminuido, sed aumentada, sudoración aumentada, peso disminuido.

Al examen clínico se encontró; PA: 105/65, FC: 100, FR:20, T $39^{\circ}$ C. En MEG, REN, hidratado agudamente enfermo, con palidez severa de piel y mucosas, leve ictericia en escleras. Linfoadenomegalia posterior cervical. Tórax y Pulmón: N, Hígado aumentado de tamaño a $3 \mathrm{~cm}$ drcd, traube ocupado. Fondo de ojo: Hemorragias retinianas con algunos infiltrados blanquecinos en ambos ojos (ver foto 1).

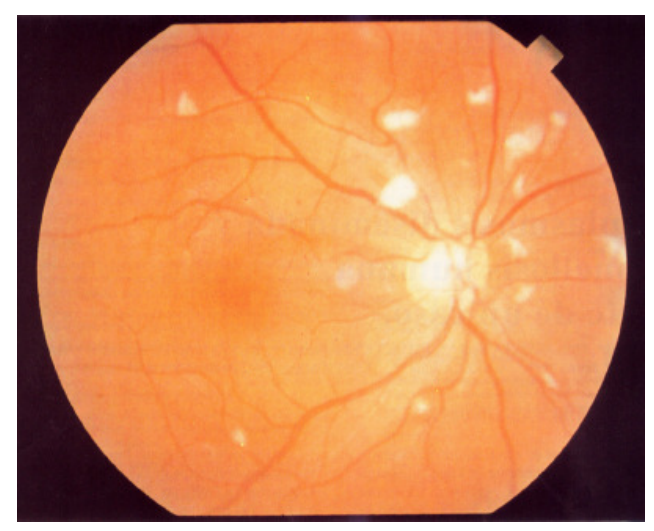


De los análisis se tiene. Hematocrito, en $20 \%$ con índice de producción de 0.7 y reticulocitos de $7.3 \%$, leucocitos en 5600 , con $0 \%$ de bastones y linfocitos en $17 \%$, las pruebas hepáticas al inicio fueron normales para luego incrementarse la fosfatasa alcalinay las bilirrubinas, manteniéndose normales las transminasas. El frotis sanguíneos fue positivo para bartonella $(<10 \%)$, negativo para plasmodium, Elisa para HIV (-) IFI para Toxoplasma (-), elisa para CMV ( - ), hemocultivo x 4 (-), mielocultivo x 1 (-). En los cultivos (3 hemocultivos y 1 mielocultivo) en agar Sabouraud se aisló H. capsulatum. En una biopsia hepática y en un coágulo de médula ósea se encontró granulomas con presencia del H. capsulatum (ver foto 2 de biopsia hepática).

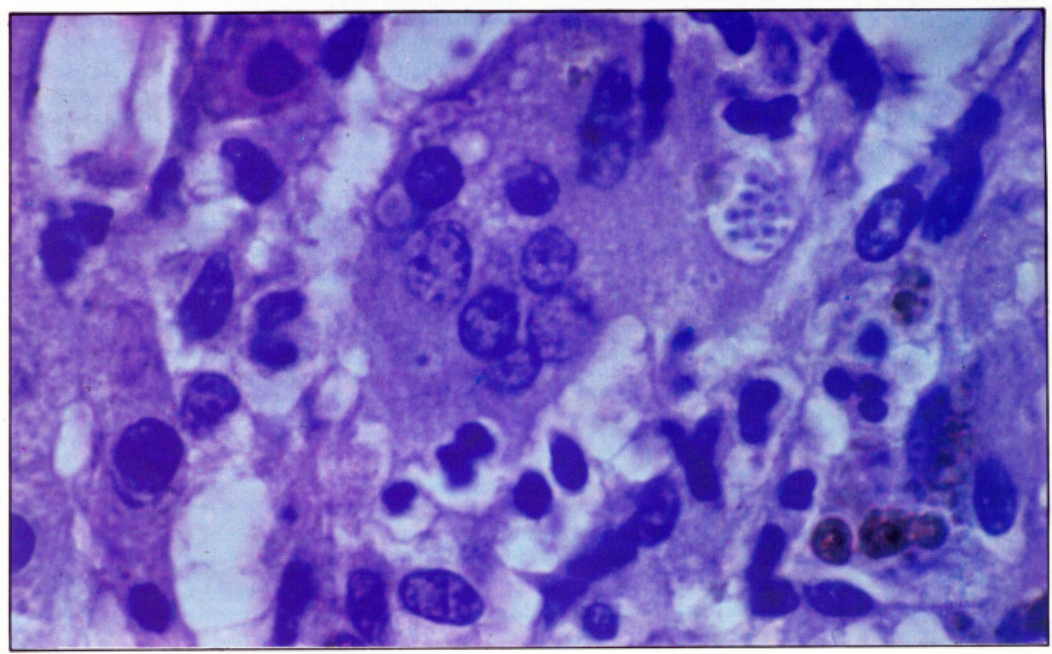

El paciente recibió 6 transfusiones de paquetes globulares y CAF oral por catorce días, a pesar de ello evolucionó con persistencia de la fiebre, mejorando sólo ligeramente las molestias iniciales, al incrementarse la fosfatasa alcalina en forma importante, se le practicó biopsia hepática, en la cual se evidenciaron las levaduras compatibles con $\mathrm{H}$. capsulatum, este diagnóstico fue corroborado con los cultivos posteriores. Por ello recibió tratamiento base de itraconazol a la dosis de $200 \mathrm{mg}$ por día con lo cual la fiebre empieza a disminuir así como las otras molestias, saliendo de alta mejorado.

\section{DISCUSION}

La enfermedad de Carrión o Bartonellosis Humana, enfermedad histórica de la medicina peruana $(1,2)$, es una enfermedad que se presenta predominantemente en el Perú, en especial en algunos valles interandinos, abarcando desde el norte del Perú (San Ignacio en Cajamarca) hasta el sur (San Juan de Huancavelica) y el centro (Valle del Mantaro) (3).

En la era preantibiótica la fase aguda febril anemizante tenia una gran mortalidad y llegaba hasta a más de $40 \%$, entre las complicaciones asociadas, se señalaban a la Tuberculosis, salmonellosis (S.dublin, S. anatum,S.enteriditis, S. tiphy), amebiasis, etc (4) . Con la aparición de los antibióticos y otras medidas como las transfusiones de glóbulos rojos(paquete globular) la mortalidad disminuyó en forma importante, pero aún hoy día sigue siendo alta, así en la serie del Hospital Nacional Cayetano Heredia la mortalidad ha sido de un $8 \%$ en pacientes en fase aguda $(5,6,7)$. En los últimos años se 
ha reconocido que la complicación neurológica está asociada a una mayor mortalidad (8).

La enfermedad de Carrión en sus dos fases se parece en algo al Síndrome de Inmunodeficiencia Adquirida. Así para la fase aguda se ha descrito desde épocas antiguas y también ahora último (9) una depresión transitoria de la Inmunidad Celular.

Para la fase eruptiva hay nuevos estudios que demuestran semejanzas con el SIDA, así algunos autores describen lesiones denominadas recientemente como "Angiomatosis bacilar", la que fué inicialmente descrita en pacientes con SIDA (10) y semeja en muchos aspectos a la Verruga Peruana, tanto por la presencia de bacterias como por la respuesta histológica. El agente causal de esta enfermedad es hasta la fecha desconocido.

El estado actual de las investigaciones sobre la Verruga Peruana revela por ejemplo que la bacteria produciría un factor angiogenético que, estimularía la proliferación de células endoteliales (11). Los Drs. Arrese y Pierard en estudios inmunohistoquímicos (12) han identificado una nueva población celular dérmica, los dendrocitos, que se encuentran en estrecha relación anatómica con las células endoteliales, formando parte de la unidad microvascular dérmica.

En el Hospital Nacional Cayetano Heredia (HNCH) a fin de conocer mejor la enfermedad desde hace varios años venimos estudiando en forma prospectiva a todos los pacientes con la Enfermedad de Carrión. Hasta 1991 en 52 pacientes con la fase aguda hemos tenido la ocasión de describir como infecciones asociadas: 3 pacientes con toxoplasmosis ( 1 con miocarditis fallecido), 4 pacientes con salmonellosis ( 1 tífica y 3 no tíficas), 1 sepsis a Shigella dysenteryae, etc. Asimismo tuvimos algunos pacientes libres de la bartonella que persistieron con fiebre prolongada, algunas de más de un mes de evolución en la que no se pudo averiguar la causa, pero que desaparecieron sin tratamiento alguno $(7,13)$.

El presente caso, es el primero en su género se trata de una complicación que describimos por primera vez a nivel Nacional y probablemente Mundial, es decir la presencia de histoplasmosis diseminada, complicando la enfermedad de Carrión. El paciente adquiere primero la enfermedad de Carrión y hace posteriormente una histoplasmosis diseminada al viajar a la zona endémica de Tingo María, estando en ese momento en la fase febril de la Bartonellosis, la que se caracteriza por una depresión de la inmunidad celular. El cuadro clínico de está complicación micótica fue la fiebre prolongada (sin tener bartonellas en sangre), con compromiso hepático y localización en médula ósea, pudiendo cultivarse de este sitio y de la sangre el Histoplasma capsulatum. En 1990 la Dra. Bustamante (14) en el Hospital Nacional Cayetano Heredia describió por primera vez en el Perú un caso de histoplasmosis diseminada en un paciente también procedente de la selva central, y que padeció SIDA, el cual tuvo una evolución tórpida, que lo llevó al exitus letalis.

Como se sabe, la Histoplasmosis es una enfermedad ligada al trastorno de la inmunidad celular, conocida antiguamente como Enfermedad de Darling quien la describió en 1905 (15). En 1935 el Dr. Weiss publicó algunos casos de enfermedades respiratorias ligadas a la histoplasmosis, bautizándolas como la "Fiebre de Tingo María" (16). En 1955 se 
realizan varios estudios con histoplasmina y estudios radiológicos que revelan la presencia de la enfermedad en Tingo María, Pucallpa, Iquitos, etc. En los últimos años se ha reportado la presencia de la histoplasmosis en el valle del río Santiago (17) y en el Valle de Palcazo (Selva Central) (18).

La Histoplasmosis tiene una serie de formas clínicas que van desde la forma asintomática (la más frecuente), a la forma pulmonar primaria benigna, la forma pulmonar crónica, la forma diseminada aguda (la que tuvo el paciente) y la forma diseminada crónica (15). Desde 1981 al aparecer el SIDA, esta última forma de Histoplasmosis diseminada se ha reportado en forma importante en los EE.UU. La mortalidad ha sido alta llegando en algunas series hasta el 100\% $(19,20)$.

Si bien es cierto que el Anfotericin B es una droga que ha sido útil en el tratamiento de esta micosis, en los últimos años se están usando nuevas terapias orales a base de los derivados imidazólicos como el Ketoconazol, fluconazol e itraconazol. El itraconazol es un triazol muy efectivo en todas las formas de histoplasmosis que requieran tratamiento, con la excepción de aquellos con compromiso meníngeo (21). El paciente del presente reporte, recibió $200 \mathrm{mg} /$ día de itraconazol y mostró una rápida mejoría clínica con disminución de la curva febril, sin embargo no se pudo hacer una evaluación completa porque el paciente no acudió a sus controles clínicos.

En los pacientes con fiebre de origen oscuro, en las series internacionales, $(22,23,24)$ se ha considerado a la hepatitis granulomatosa (infecciosa y no infecciosa) como una de sus causas; también a la histoplasmosis diseminada se la ha señalado como causante de fiebre persistente. En cambio en la serie de Fiebre de origen oscuro del Hospital Cayetano Heredia (25) no se la había incluido al no encontrar ningún caso. El paciente que presentamos reunía los criterios para considerar FOO (22), tenía más de tres semanas (26 días) sin precisar la causa de la fiebre, la fiebre era mayor de $\left(38.3^{\circ} \mathrm{C}\right)$ y todos los análisis básicos (Rx Torax, cultivos, búsqueda de Bacilo de Koch en varios sitios, etc.) eran negativos.

De ahí que el hallazgo de H. capsulatum en la biopsia hepática nos llamó a sorpresa, con la confirmación del diagnóstico en los cultivos y la presencia del hongo en la médula ósea, se llegó al diagnóstico correcto. Asimismo destacamos la importancia del antecedente epidemiológico para el enfoque diagnóstico, el que nos sirvió para diagnosticar en primer lugar la enfermedad de Carrión y en segundo lugar la histoplasmosis. Muchas veces no se pregunta la procedencia de los pacientes, lugares que visitaron, etc. Inclusive en nuestro estudio sobre la enfermedad de Carrión (7) cerca de un $13 \%$ de los pacientes que procedían de las zonas endémicas al ingresar al $\mathrm{HNCH}$, los médicos que vieron a los pacientes no plantearon en el diagnóstico diferencial a la enfermedad de Carrión.

Una vez más la enfermedad de Carrión cobra importancia, no sólo por lo parecido de su perfil clínico en relación al SIDA, sino por lo llamativo de sus complicaciones, muchas nuevas, no descritas anteriormente y otras que no se conocen y que requerirán futuros estudios.

\section{REFERENCIAS BIBLIOGRÁFICAS}

1.Maguiña C, Maguiña T, Arrese E, Pierard G. La Verruga Peruana Chez les Huaylas. Rev Eur Derm 1990; 2: 594-596. 
2.García U, García F. Bartonellosis. Am J Clin Pathol. 1991; 95: 58-66.

3.Maguiña C, Pérez E. La Enfermedad de Carrión y Leishmaniasis andina en la región de Conchudos. Diagnóstico1985; 16: 5-12.

4.Cuadra M. Salmonellosis complication in human bartonellosis. Texas report of Biological Medicine 1956; 14: 97-100.

5.Maguiña $\mathrm{C}$, Lumbreras $\mathrm{H}$, et al. Estudio clínico y de laboratorio de 31 pacientes en fase aguda de la enfermedad de Carrión. Cuarta Jornadas Científicas UPCH, 1986.

6.Maguiña C, Lumbreras H, Gotuzzo E, Crosby E, Irrivaren J. Clinical and laboratory study of patients with acute phase of Carrión's disease in the HCH between 19691987. Rio de Janeiro: Internacional Congress for Infectious Disease, 1988; 63.

7.Maguiña C, Gotuzzo E. Verruga Peruana o Enfermedad de Carrión, Libro Pediatría Meneghelloo, Chile. Cuarta edición 1991; 1: 696-697.

8.Acosta R. Neurobartonellosis en Lima. Tesis Bachiller en Medicina. Universidad Peruana Cayetano Heredia. 1988.

9.Patrucco E. Estudio de parámetros inmunológicos en pacientes con bartonellosis humana. Diagnóstico 1983; 12:138-144.

10.Leboit P. Berger T. Bacillary angiomatosis. The histopathology and differential diagnosis of a pseudoneoplastic infection in patients with human inmunodeficiency virus disease. Amer Jour of Surg Patho 989; 13: 909-920.

11.García F, et al. Bartonella bacilliformis stimulates endothelial cells in vitro and is angiogenic in vivo. Am J Pathol 1990; 136: 1125-1134.

12. Arrese Estrada J, Pierard G. Factor XIIa positive dendrocytes and the dermal microvascular unit. Dermatologica 1990; 180: 51-53.

13.Maguiña C, Gotuzzo E. La Enfermedad de Carrión. Enf Inf Micr Clin 1988; 6: $432-$ 435.

14. Bustamante B, Seac C. Histoplasmosis diseminada en SIDA. Reporte de un caso. Rev Med Hered 1991; 1: 51-52.

15.Goodwin R and Des Perez R. Histoplasma Capsulatum in Mandell G,Douglas G and Benneth. Principles and practice of Infectious Disease. Second edition, 1985; 14681476.

16. Medicina tropical UNMSM. Folleto 1988. Lecciones de Histoplasmosis. Historia, Clínica y Terapéutica.

17.Bustamante B y Llanos A. Estudio epidemiológico con histoplasmina en la región del Rio Santiago. Cuartas Jornadas Científicas de la UPCH, 1986.

18.Bustamante B y Chang J. Estudio epidemiológico con histoplasmina en el Valle del Palcazo. Quintas Jornadas Científicas, UPCH. 1988.

19.Graybill J. Histoplasmosis and AIDS. Journ of Infec Dis. 1988; 1953:623-6.

20.Goodwin R. et al. Disseminated histoplasmosis Clinical and pathologic correlations. Medicine 1980; 59: 1-4.

21.Negroni R. Recent advances in Systemic antifungal therapy. Histoplasmosis. Proceedings X Congress of the International Society for human and animal micology. Spain, 1988.

22.Petersdof R. Fever of undetermined origin. Medicine 1961; 40:1.

23.Larson E. Fever of undetermined origin. Medicine 1962; 61: 269-292.

24.Petersdof R. Fever of unknown origin. Ann Intern Med. 1969: 70: 864-869.

25.Gotuzzo E. Comunicación personal. 